\title{
Hemolysis in children with Glucose-6-Phosphate Dehydrogenase Deficiency after ingestion of fava beans; facts predicting severity
}

\author{
Hasanein H. Ghali', Doaa Alem Al Mamoori²
}

'Department of Pediatrics, College of Medicine, University of Baghdad, Children Welfare Teaching Hospital, Baghdad Medical City, Baghdad, Iraq.

${ }^{2}$ Department of Emergency Medicine, Baghdad Medical City, Baghdad, Iraq.

Corresponding Author: Hasanein H. Ghali (E-mail: hasaneinghali@comed.uobaghdad.edu.iq)

(Submitted: 02 September 2020 - Revised version received: 24 September 2020 - Accepted: 26 - October 2020 - Published online: 26 December 2020)

\begin{abstract}
Objectives: This study aimed to assess the demographic, clinical, and biochemical characteristics as predictors of hemolysis after ingesting fava beans.

Methods: A cross-sectional study was undertaken. A total of 57 patients with Glucose-6-Phosphate Dehydrogenase (G6PD) deficiency were recruited from the Emergency Department of Children Welfare Teaching Hospital, Medical City. Data were collected using a specially designed form. A purposive sampling method was used to recruit 57 patients (49 males and 8 females) who were visiting the pediatric emergency department from March to May 2017 with a presentation of acute hemolytic episode of G6PD. Patients were classified into mild or severe hemolysis groups based on their hemoglobin level at the time of admission.

Results: Younger age group patients tend to present with the severe form of hemolysis (3.59 years with a $P$ value of 0.001 ). No significant gender susceptibility between both types of hemolysis. The urban area-based living individuals tend to present with mild hemolysis while those from rural areas tends to present with more severe episodes of hemolysis ( $P$ value 0.001 ). There was a significant correlation between the type of fava bean ingestion (fresh or dried) and the severity of hemolysis, those who presented with more severe hemolysis usually had a history of ingestion of fresh type of fava bean. Eight individuals of severe type recorded previous episodes of hemolysis while three individuals of the mild type recorded previous episodes ( $P$ value 0.001 ). Family history of G6PD was significant in $88.2 \%$ of individuals with the severe form ( $P$ value 0.005).

Conclusion: This study is aimed to report several factors that might predict the severity of hemolysis among patients with G6PD deficiency. Younger age, residence in a rural region, ingestion of fresh fava beans, and history of frequent hemolysis incidents are predictors of developing severe hemolysis among children admitted to the emergency room of Children Welfare Teaching Hospital in Medical City/ Baghdad.

Keywords: Favism, G6PD deficiency, severe haemolysis, Iraqi children
\end{abstract}

\section{Introduction}

Glucose-6-phosphate dehydrogenase (G6PD) deficiency is known as a disorder transmitted by an x-linked enzymatic mutation in the G6PD gene. It has been known that G6PD is the most common human enzymatic defect, represented in around 400 million humans around the world. The enzyme catalyzes pentose phosphate pathway's first reaction, offering antioxidant protection to the cells counteracting any oxidative stress. Hence, individuals with deficiency of this enzyme are unable to protect red blood cells (RBC) against myriad of oxidative stresses. ${ }^{1}$ The most recent worldwide assessment of the disease prevalence had shown that the Middle Eastern region stands second in prevalence (7.2\%). ${ }^{2}$ G6PD deficiency is common among Iraqis; with an estimated prevalence around $6 \%$ in the middle part (Baghdad), ${ }^{3}$ and $10.9 \%$ in northern Iraq. ${ }^{4}$ The disease is manifested silently, unless an affected individual eats fava beans or takes some hemolysis-inducing medicines. ${ }^{5}$ The presentation of this disease depends mainly on the degree of deficiency and the severity of hemolytic attack. ${ }^{1}$ It presents usually with a sudden episode of acute hemolysis including fatigue, pallor, and dark colored urine. ${ }^{6} \mathrm{G} 6 \mathrm{PD}$ deficiency represents a major health problem in Iraq; particularly during the harvest of fava beans, which peaks during spring season. Moreover, the diagnosis of G6PD deficiency continues to be a challenge for the health department, especially in regions where diagnostic facilities are not available and growing fava beans are widespread.

This study was conducted to study the profile of G6PD and to examine the presence of any demographic, clinical, or biochemical factors that may be associated with the severity of hemolysis in patients treated at Emergency room of Children Welfare Teaching Hospital in Medical City/Baghdad.

\section{Patients and Methods}

\section{Patients and Design}

This cross-sectional study was conducted at the emergency room of Children Welfare Teaching Hospital in Medical City, Baghdad, Iraq. This hospital is a tertiary center and one of the largest hospitals in Baghdad with over 250 beds for patients. A purposive sampling method was used to recruit 57 patients who were visiting the pediatric emergency department from March to May 2017 with a presentation of acute hemolytic episode of G6PD.

This study included patients who are younger than 14 years, were previously diagnosed with G6PD deficiency via G6PD enzymatic assay, and had a history of ingestion of fava beans or with the first presentation of a hemolytic 
episode of the disease. The medical diagnosis of G6PD deficiency was confirmed according to the medical history, clinical presentation at the time of admission and/or laboratory investigations. Patients presented with sudden onset of pallor, jaundice, dark colored urine, with features of hemolysis (increased bilirubin, low hemoglobin, increased reticulocyte count if available, and increased urinary urobilinogen if available) were further investigated to exclude other hereditary hemolytic disorders and/or acquired autoimmune hemolytic anemia. Those patients with suspicious blood film results and negative Coombs test results were asked to do a G6PD enzymatic assay after hemolysis resolves. Because the G6PD enzyme can be falsely normal during a hemolysis episode, the enzymatic activity is usually assessed 1-3 months after a crisis for those with negative history of diagnosis. Information about recent infection or ingestion of drugs that may induce hemolysis was also elicited. Patients who had ingested these drugs were excluded. The legal guardians of eligible patients were approached and invited to participate in this study. In accordance with the Helsinki Declaration of ethical principles, ${ }^{7}$ informed verbal consent was obtained from the legal guardians before enrolment in this study.

\section{Data Collection and Quantification}

Relevant demographic information about the patients were elicited, including age, gender, and residence (urban, or rural). Clinical data involved medical history and physical examination. Data about the form of fava bean ingested (fresh or dried), duration of exposure to fava beans before admission (in days), duration of onset of symptoms before admission (in days), presence of vomiting during the attack, history of previous episodes of hemolysis, history of neonatal jaundice, and personal or family history of G6PD deficiency were recorded. Information from the physical examination included fever (defined as temperature $\geq 38^{\circ} \mathrm{C}$ axillary corrected), pallor, jaundice, and organomegaly. Data about hematological and biochemical investigations that were performed at admission included blood group (A, B, AB or $\mathrm{O}), \mathrm{Rh}$ (negative or positive), hemoglobin level, white blood cell (WBC) count, platelets, total serum bilirubin (TSB), reticulocyte count, alanine aminotransferase (ALT), and aspartate aminotransferase (AST). Data were quantified and expressed as either frequencies (for categorical variables) or means and standard deviation (for continuous variables). The severity of hemolysis was assessed based on hemoglobin level at admission. Cases with a hemoglobin level $\leq 5 \mathrm{~g} / \mathrm{dL}$ were considered severe, while patients with a hemoglobin level of more than $5 \mathrm{~g} / \mathrm{dL}$ were classed as having mild-moderate hemolysis.

\section{Statistics}

Descriptive analysis was performed to describe the study sample. The differences between the mild and severe hemolysis groups were assessed by bivariate analyses. These included independent samples $t$-test for continuous data and Chi square analysis for categorical data. Because the outcome variable is dichotomous (mild vs severe hemolysis). All analyses were performed using IBM SPSS (SPSS, Chicago, IL, USA) Statistics for mac.

\section{Results}

A total of 57 were recruited in this study. Of 57 children with G6PD deficiency, 17 (29.8\%) were diagnosed as severe cases depending on the level of hemoglobin at time of presentation to the emergency room in Children Welfare Teaching Hospital.

The total number is 57 patients. The mean age is 4.35 years, males were constituting $85.9 \%, 32$ patients were living in Urban area mostly from Baghdad, two-thirds of the patients have recorded ingestion of fresh type of fava beans (64.9\%), while one-third ingested dried type (35\%). Ten patients were already diagnosed with G6PD prior to the presentation; six of them were diagnosed based on previous episodes of hemolysis, and four depending on routine assessment for a suspected family member.

Analysis of the demographic and clinical characteristics of the 57 patients according to the severity of hemolysis (mild vs severe) and the results of the independent samples $t$-test (for continuous variables) and Chi square (for categorical variables) are represented in Table 1. Younger age group patients tend to present with the severe form of hemolysis (3.59 years with a $P$ value of 0.001 ). No significant gender susceptibility was present between both types of hemolysis. The urban areabased living individuals tend to present with mild hemolysis while those from rural areas tend to present with more severe episodes of hemolysis ( $P$ value 0.001$)$. Moreover, there was a significant correlation between the type of fava bean ingestion and the severity of hemolysis, those who presented with more severe hemolysis usually had a history of ingestion of fresh type of fava bean.

The mean duration of exposure was longer in those with mild hemolysis (2.87 days), and the mean onset of symptoms was also longer in severe hemolysis (2.20 days) with a significant $P$ value of 0.005 . Eight individuals of severe type recorded previous episodes of hemolysis while three individuals of the mild type recorded previous episodes ( $P$ value 0.001 ). Eightytwo percent of the severe cases recorded history of neonatal jaundice early in life treated with phototherapy, or exchange transfusion while $35 \%$ of the mild type recorded previous neonatal episode of jaundice ( $P$ value 0.001 ).

Family history of G6PD was significant in $88.2 \%$ of individuals with the severe form ( $P$ value 0.005$)$. No significant correlation was seen between the type of hemolysis and the presence of vomiting, fever, jaundice, organomegaly, or pallor in those patients. Pallor and jaundice detection in this study depend mainly on the clinical findings explored by the physician who was in charge in the ER.

Baseline laboratory characteristics of 57 children with the diagnosis of G6PD had shown the following results: blood group was distributed as following: group $\mathrm{O}$ was detected in $43.8 \%$ of patients, group B was detected in $26.3 \%$, this is followed by group $\mathrm{A}$ in $22.8 \%$, and $\mathrm{AB}$ in $7 \%$. Rh positive was seen in 50 patients. The $\mathrm{CBC}$ profile showed the mean hemoglobin level detected was $6.1 \mathrm{~g} / \mathrm{dl}$, ranging from 2.1 to $8.5 \mathrm{~g} / \mathrm{dl}$, the mean reticulocyte count was $8 \%$ ranging from 3.5 to $25 \%$. Liver function tests (TSB, ALT, and AST) were carried out to all patients where the mean serum bilirubin detected is 3.88 $\mathrm{mg} / \mathrm{dl}$, ranging from 1.9 to $9.5 \mathrm{mg} / \mathrm{dl}$. Table 2 shows the analysis of the laboratory characteristics of the 57 patients according to the severity of hemolysis (mild vs severe) and the results 


\begin{tabular}{|c|c|c|c|}
\hline Item & Mild hemolysis $(n=40)$ & Severe hemolysis $(n=17)$ & $P$ value \\
\hline Age (years)(SD) & $5.57(2.11)$ & $3.59(1.98)$ & 0.001 \\
\hline \multicolumn{4}{|l|}{ Gender } \\
\hline Male & $34(85.0 \%)$ & $15(88.20 \%)$ & \\
\hline Female & $6(15.0 \%)$ & $2(11.76 \%)$ & 1 \\
\hline \multicolumn{4}{|l|}{ Residence } \\
\hline Urban & $28(70.0 \%)$ & $4(23.5 \%)$ & \\
\hline Rural & $12(30.0 \%)$ & $13(76.4 \%)$ & 0.001 \\
\hline \multicolumn{4}{|l|}{ Form of fava beans } \\
\hline Fresh & $15(37.5 \%)$ & $12(70.5 \%)$ & \\
\hline Dried & $25(62.5 \%)$ & $5(29.4 \%)$ & 0.04 \\
\hline \multicolumn{4}{|l|}{ History } \\
\hline Mean Duration of exposure(SD) & $2.87(1.69)$ & $2.50(1.45)$ & 0.1 \\
\hline Mean Onset of symptoms(SD) & $1.42(0.51)$ & $2.20(1.01)$ & 0.005 \\
\hline Previous episodes & $3(7.5 \%)$ & $8(41.1 \%)$ & 0.001 \\
\hline History of neonatal jaundice & $14(35.0 \%)$ & $14(82.3 \%)$ & 0.001 \\
\hline G6PD cases & $7(17.5 \%)$ & $2(17.6 \%)$ & 0.9 \\
\hline Family history of G6PD & $15(37.5 \%)$ & 15 (88.2\%) & 0.005 \\
\hline \multicolumn{4}{|l|}{ Clinical characteristics } \\
\hline Presence of vomiting & $15(37.5 \%)$ & $5(29.4 \%)$ & 0.1 \\
\hline Presence of fever & $17(42.5 \%)$ & $6(35.2 \%)$ & 0.7 \\
\hline Presence of pallor & $36(90.0 \%)$ & $17(100.0 \%)$ & 0.3 \\
\hline Presence of clinical jaundice & 37 (92.5\%) & $17(100.0 \%)$ & 0.5 \\
\hline Presence of organomegaly & $1(2.5 \%)$ & $1(5.8 \%)$ & 0.5 \\
\hline
\end{tabular}

of the independent samples $t$-test (for continuous variables) and Chi square (for categorical variables). No significant correlation was detected between both types of hemolysis and blood grouping or Rh type. Significant correlation between hemoglobin level and the severity of hemolysis is expected to be detected as the classification of hemolysis was mainly based on the hemoglobin level. Additionally, TSB level and reticulocyte count were found to be significantly higher in the severe form type (TSB mean of $5.07 \mathrm{mg} / \mathrm{dl}$ and reticulocytes count of $11.9 \%$ with $P$ values of 0.02 and $<0.001$, respectively). There was no statistical difference in the level of platelets and WBCs between both types of hemolysis.

\section{Discussion}

This report discusses the relationship between demographic profile, clinical, hematological and biochemical characteristics, and the severity of hemolysis among favic patients. The results of this study showed that patients with severe hemolysis were younger as compared to mild cases. This is consistent with a previous study conducted in Jordan, which reported similar results. ${ }^{8}$ Although the mechanism for this relationship is not clear, patients with more severe deficiency in the G6PD enzyme tend to present at earlier age in the course of the disease. Because acute hemolysis in favic patients is triggered by ingestion of fava beans, younger children may not be intellectually able to realize the impact of ingesting fava beans, especially if unsupervised by their parents. But this is not the case here, as those who already knew that they are G6PD-deficient are three patients only in the severe form.

Despite the fact that the results of the present study revealed that the majority $(85.9 \%)$ of patients are male, the gender difference between mild and severe hemolysis groups was not significant. Male predominance in patients with G6PD deficiency was widely reported. ${ }^{9-11}$ Because G6PD deficiency follows an X-linked pattern of inheritance, it is fully expressed in heterozygous males and homozygous females. ${ }^{12}$ Therefore, heterozygous females are usually asymptomatic.

The results also revealed that in comparison to city living, favic patients living in rural areas were more likely to develop severe hemolysis. Several factors may render people living in rural areas more susceptible to the severe form of hemolysis. First, the health beliefs in rural areas are still primitive, especially when it comes to disease and illness. ${ }^{13}$ Second, there is a lack of health awareness and education in the regional area regarding the nature of the disease and the factors that may 


\begin{tabular}{|c|c|c|c|}
\hline Item & Mild hemolysis $(n=40)$ & Severe hemolysis $(n=17)$ & $P$ value \\
\hline \multicolumn{4}{|l|}{ Blood group } \\
\hline 0 & $17(42.5 \%)$ & $8(47.0 \%)$ & \\
\hline B & $10(25.0 \%)$ & $5(29.4 \%)$ & \\
\hline A & $9(22.5 \%)$ & $4(23.5 \%)$ & \\
\hline$A B$ & $4(10.0 \%)$ & $0(0.0 \%)$ & 0.6 \\
\hline \multicolumn{4}{|l|}{$\mathbf{R h}$} \\
\hline Positive & $35(87.5 \%)$ & $15(88.2 \%)$ & \\
\hline Negative & $5(12.5 \%)$ & $2(11.7 \%)$ & 1 \\
\hline \multicolumn{4}{|c|}{ Hematological parameters } \\
\hline Haemoglobin g/dL(SD) & $7.05(1.22)$ & $4.46(0.58)$ & 0.001 \\
\hline$W B C \times 10^{9} / L(S D)$ & $4.90(3.51)$ & $5.78(4.40)$ & 0.4 \\
\hline Platelets x 109\%/L(SD) & $165.45(81.16)$ & $184.86(132.63)$ & 0.5 \\
\hline TSB, mg/dL(SD) & $3.09(1.80)$ & $5.07(0.95)$ & 0.02 \\
\hline Reticulocytes, \%(SD) & $6.92(4.88)$ & $11.91(5.56)$ & 0.001 \\
\hline \multicolumn{4}{|l|}{ Biochemistry } \\
\hline TSB, mg/dL(SD) & $3.09(1.80)$ & $5.07(0.95)$ & 0.02 \\
\hline ALT, IU/L(SD) & $39.81(17.91)$ & $47.19(11.51)$ & 0.4 \\
\hline AST, IU/L(SD) & $23.39(22.41)$ & $25.65(19.81)$ & 0.19 \\
\hline
\end{tabular}

ALT: alanine aminotransferase; AST: aspartate aminotransferase; TSB: total serum bilirubin; WBC: white blood cells. All baseline and laboratory values are expressed as mean \pm standard deviation (SD) except as indicated otherwise.

contribute to inducing hemolysis. ${ }^{13}$ Parents may not be aware about the role of fava beans in inducing hemolysis among their children, even if they are not diagnosed with G6PD, and even if there is family history of G6PD, people from urban areas tend to avoid ingesting fava bean. Third, regional areas in Iraq are well-known for growing fava beans, unlike the urban region. The higher exposure to fava beans in rural areas, especially the fresh form during the harvest season, may explain the higher probability of developing severe hemolysis. This was also evident in this study as patients who had consumed fresh beans had more severe hemolysis as compared to those who had consumed dried beans. This is consistent with several reports from other Middle-Eastern countries, which showed that seasonal peaks of favism are corresponding to harvesting fresh fava beans. ${ }^{14,15}$ The frequency of previous episodes of hemolysis could predict the severity of hemolysis in this study. Patients with a history of more frequent hemolysis incidents had more severe hemolysis. The same was found for those who had a history of neonatal jaundice. The frequency of previous incidents may reflect the level of G6PD enzyme activity. According to the WHO classification of G6PD deficiency, the severity of G6PD enzyme deficiency corresponds to the chronicity of hemolysis. ${ }^{16}$ Excessive heme metabolism resulting from severe intravascular hemolysis leads to significant increases in bilirubin, which overwhelms the hepatic conjugation process resulting in unconjugated hyperbilirubinemia state. ${ }^{17}$ Biochemically, our results showed that the more increase in TSB, and reticulocyte count could predict the severity of hemolysis. Owing to intravascular hemolysis and destruction of RBCs, increased breakdown of hemoglobin results in increased TSB. ${ }^{18}$ As the bone marrow attempts to compensate for the continuous RBC destruction, reticulocytes release into peripheral blood drastically increases, reflecting the increasing amount of erythropoiesis. ${ }^{19}$ This study has several noteworthy limitations. First, part of the data were collected based on patients' and parents' recall. Therefore, recall bias might have been present. Second, study participants were recruited using non-probability sampling, which has a limited generalizability. ${ }^{20}$ Therefore, these findings should be interpreted with caution, as it does not represent the entire Iraqi population. In addition, the clinical assessment of patients was carried out by physicians, which can be subjective. Therefore, measurement bias might have been present as well.

\section{References}

1. Cappellini MD, Fiorelli G. Glucose-6-phosphate dehydrogenase deficiency. Lancet (London, England), 2008;371(9606):64-74

2. Nkhoma ET, Poole C, Vannappagari V, Hall SA, Beutler E. The global prevalence of glucose-6-phosphate dehydrogenase deficiency: A systematic review and meta-analysis. Blood Cells Mol Dis, 2009:42(3):267-278.

3. Al-Musawi BM, Al-Allawi N, Abdul-Majeed BA, Eissa AA, Jubrael JM, Hamamy H. Molecular characterization of glucose-6-phosphate dehydrogenase deficient variants in Baghdad city - Iraq. BMC Blood Disord. 2012;12:4.

4. Al-Musawi, B.M., Al-Allawi N., Abdul-Majeed, B.A. et al. Molecular characterization of glucose-6-phosphate dehydrogenase deficient variants in Baghdad city - Iraq. BMC Hematol, 2012;12:4

5. Merdin A, Avci F, Guzelay N,Glucose-6-phosphate dehydrogenase deficiency presented with convulsion: A rare case. Hematol Rep, 2014;6(1):5266. 
6. Frank JE. Diagnosis and management of G6PD deficiency. Am Family Physician, 2005;72(7):1277-1282

7. World Medical Association Declaration of Helsinki. Ethical principles for medical research involving human subjects, JAMA, 2013;310(20):2191-2194

8. Al-Sweedan SA, Jdaitawi H, Khriesat WM, Khader YY, Al-Rimawi HS. Predictors of severe hemolysis in patients with glucose-6-phosphate dehydrogenase deficiency following exposure to oxidant stresses. Hematol/ Oncol Stem Cell Ther. 2009;2(2):354-357.

9. Yao LQ, Zou TB, Wang XT, Quan X, Chen Q, Yang FB, Hu LS, Fan LM, Wang M, Feng XY, Liu JT, Zhao ZM [G6PD deficiency among children under 7 years old from Yunnan with unique ethnic minority origin]. Chin J Med Genet. 2013;30 (2):189-194.

10. Von Fricken ME, Weppelmann TA, Eaton WT, Alam MT, Carter TE, Schick $L$, Masse R, Romain JR, Okech BA, Prevalence of glucose-6-phosphate dehydrogenase (G6PD) deficiency in the Ouest and Sud-Est departments of Haiti. Actatropica. 2014;135:62-66.

11. Ntaios G, Chatzinikolaou A, Tomos C, Manolopoulos C, Karalazou P, Nikolaidou A, Alexiou-Daniel S, Prevalence of glucose-6-phosphate dehydrogenase deficiency in Northern Greece. Intern Med J. 2008;38(3):204-206.Guindo A, Fairhurst RM, Doumbo OK, Wellems TE,
Diallo DA ,X-linked G6PD deficiency protects hemizygous males but not heterozygous females against severe malaria. PLoS Med. 2007;4(3):66.

12. Sultan AS, Medicine in the 21st century: the situation in a rural Iraqi community. Patient Educ Counsel. 2007;68(1):66-69.

13. Belsey MA, The epidemiology of favism. Bull World Health Organ 1973:48(1):1-13.

14. Kattamis CA, Kyriazakou M, Chaidas S. Favism: clinical and biochemical data. J Med Genet. 1969;6(1):34-41.

15. Glucose-6-phosphate dehydrogenase deficiency. WHO Working Group. Bull World Health Organ. 1989;67(6):601-611.

16. Roche SP, Kobos R, Jaundice in the adult patient. Am. Family Phys. 2004;69(2):299-304

17. Tabbara IA Hemolytic anemias. Diagnosis and management. Med Clin North Am. 1992;76(3):649-668.

18. Gilmer PR, Jr., Koepke JA. The reticulocyte. An approach to definition. Am J Clin Pathol. 1976;66(1):262-267.

19. Catania JA, Dolcini MM, Orellana R, Narayanan V, Nonprobability and probability-based sampling strategies in sexual science. J Sex Res. 2015;52 (4):396-411. 